\title{
Invaginación basilar no asociada a Síndrome de Chiari y compresión crítica de unión bulbomedular: Revisión bibliográfica y técnica quirúrgica en un caso clínico
}

José Vicente Vásconez Fabre ${ }^{1}$, Mario Pinos Gavilanez², Victoria Ronquillo Campuzano², Gisella Varas Valverde ${ }^{2}$, Patricia Lara Flores², Jhony Ramos Núñez², Estuardo Reinoso Pazmiño², Xavier Páez Pesantes²

Neurocirujano Clínica Hospital del Profesor, Santiago, Chile.

2 Hospital Roberto Gilbert Elizalde, Guayaquil, Ecuador.

Rev. Chil. Neurocirugía 44: 160-166, 2018

\section{Resumen}

Introducción: La invaginación basilar es una malformación compleja de la unión cráneo cervical donde no se ha llegado a consensos de tratamiento y el enforque terapéutico debe individualizarce según el caso y las variantes anatómicas asociadas. En los casos más graves un adecuado enfoque terapéutico pude determinar regresión importante o total de la sintomatología neurológica. Objetivos: El objetivo de esta revisión es llegar al análisis de los algoritmos de tratamiento en base a una comprensión adecuada de la anatomía, variantes anatómicas y conceptualización lo más cercana posible al diagnóstico más correcto para la aplicación del mejor método de tratamiento. Métodos: Se realizó una consulta bibliográfica de los principales trabajos existentes sobre malformaciones de la unión craneocervicales colocando el énfasis en el diagnóstico anatomo morfológico, biomecánica y fisiología y radiológico. Conclusión: La adecuada conceptualización y clasificación de la patología permite tomar como marco referencial para el tratamiento con los algoritmos propuestos por Goel y Sonntag. Sin embargo, cada paciente tiene características individuales y el concepto en sí constituye un continuo de malformaciones que deberán ser resueltas por diferentes vías quirúrgicas, en diferentes tiempos si es necesario y apoyado en las diferentes tecnologías de apoyo actual como neuronavegación y seguimiento neurofisiológico trans operatorio.

Palabras clave: Invaginación basilar, Malformación ósea craneo-cervical.

\begin{abstract}
Introduction: Basilar invagination is a complex malformation of the cervicalcranial junction where tratment consensus has not been reached and the therapeutic approach must be individualized depending on the case and the associated anatomical variants. In the most severe cases, an adecuate therapeutic approach could determine significant or total regression of neurological symptoms, arrive at the analysis of the treatment algorithms based on an adecuate understanding of the anatomy, anatomycal variants and conceptualization as close as possible to the most correct diagnosis for the application of the best treatment method. Methods: A literature review of the main existing works on malformations of the craniocervical junction was made, placing emphasis on anatomical and morphological diagnosis, biomechanics and physiology and radiological findings. Conclusion: The adequate conceptualization and classification of the pathology allows to take as a reference framework for the treatment with the algorithms proposed by Goel and Sonntag. However, each patient has individual characteristics and the concept in itself constitutes a continuum of malformations that must be resolved by different surgical routes, in diferent times if necessary and supported by different currentsuport technologies such as neuronavigation and trans neurophysiological monitoring operative.
\end{abstract}

Key words: Basilar invagination, skull-cervical osseous malformation. 


\section{Introducción}

La invaginación basilar es una malformación importante en el capítulo de las anormalidades cráneo vertebrales. La asociación con malformación de Chiari y siringomielia es muy frecuente. Es importante una diferenciación morfológica clara entre platibasia, impresión basilar, invaginación basilar y retroceso odontoideo aunque todas estas alteraciones pueden ser consideradas un continuo y por lo general determinan deformidad y compresión del tronco cerebral, el problema reside en que la invaginación basilar y la platibasia se refieren a anormalidades del occipital en las que Chamberlain y otros autores utilizaron los términos indistintamente creando confusión en la conceptualización a través de los años ${ }^{1}$. La platibasia es el aplanamiento de la base del cráneo con una alteración del ángulo basal de Boogart que normalmente oscila entre 115 y $140^{\circ 1,2}$, se puede asociar a invaginación basilar lo cual puede determinar aparición de síntomas importantes. La invaginación basilar debe ser comprendida precisamente como una invaginación del contorno óseo del agujero occipital hacia el interior de la fosa posterior con reducción de sus diámetros o de su capacidad, confiriendo a la base del cráneo una forma cupular opuesta a la normal (convexobasia). Esta anómala disposición se atribuye a una sinostosis precoz de la sutura esfenooccipital ${ }^{3-4}$. La asociación de estas entidades a Síndrome de Down, Malformación de Chiari, Siringomielia y Síndrome de Klippel Feil es alta y puede determinar estrategias distintas de tratamiento. Otra patología adquirida asociada es la artritis reumatoide ${ }^{5}$.

Los síntomas de compresión de la unión cráneo cervical pueden ser inespecíficos y de difícil localización. Esto determina que el diagnóstico muchas veces puede retrasarse y ser confundido con enfermedades desmielinizantes $u$ otro tipo de patologías. Pueden desencadenarse síntomas isquémicos si hay compromiso de la arteria basilar o vertebrales, PICA o arteria espinal anterior $^{5}$. Debido a lo drástico de los síntomas y lo potencialmente invalidante o inclusive fatal el tratamiento debe ser habitualmente quirúrgico, haciendo prevalecer un objetivo y estrategia clara individualizada para cada paciente sea pediátrico o adulto, así existen varias estrategias de tratamiento como abordajes transorales, abordajes transorales extendidos lateralmente, abordajes transorales asistidos endoscópicamente y técnicas de tracción craneal asociadas.

\section{Caso clínico}

Presentamos el caso de un paciente tratado en el Servicio de Neurocirugía del Hospital Roberto Gilbert, Guayaquil, Ecuador, centro de referencia de patología neuroquirúrgica pediátrica a nivel nacional. Paciente de 17 años de edad, masculino con evolución de 5 meses caracterizada por dificultad para la marcha progresiva, debilidad en extremidades superiores y dolor occipitocervical importante de difícil manejo con analgésicos. Al ingreso presentaba debilidad en todos los grupos musculares de extremidades inferiores (2/5) e

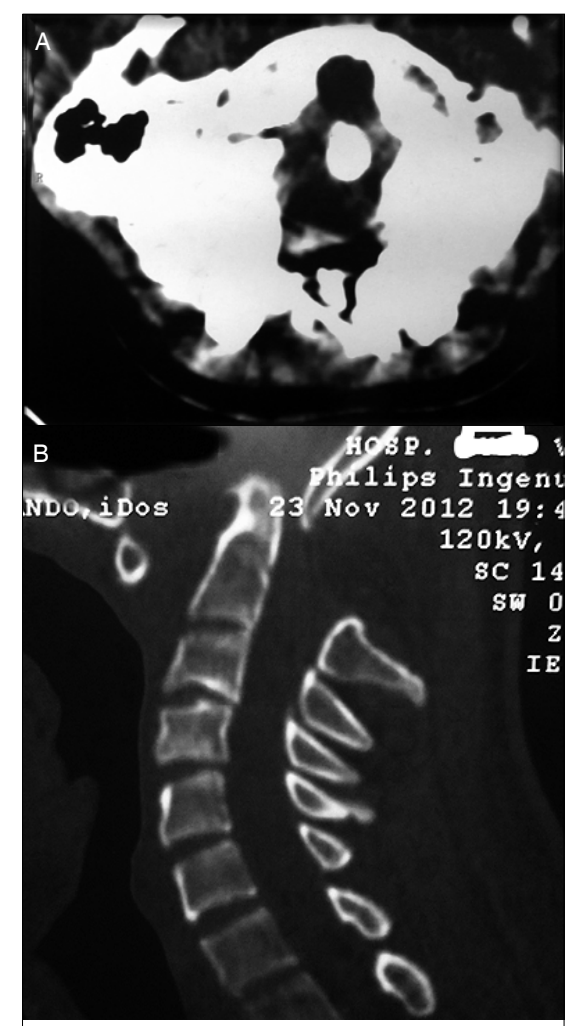

Figura 1A. TC de unión cráneo-cervical corte axial angulado para visualizar relación atlanto odontoidea que muestra posicionamiento anómalo del tope de la odontoides en el agujero magno con un aumento de la distancia atlanto odontoidea mayor de $5 \mathrm{~mm}$. Figura 1B. TC de columna cervical corte sagital que muestra la posición anómala de la odontoides contactando prácticamente el borde posterior del agujero occipital. incapacidad para la marcha, se trasladaba en silla de ruedas con asistencia, debilidad en extremidades superiores (2/5) reflejos osteotendíneos exaltados en las 4 extremidades. Abolición de reflejos cutáneo abdominales y pérdida de la sensibilidad vibratoria en extremidades inferiores. La TAC de columna cervical de ingreso mostró (Figura 1 A-B) posicionamiento anómalo de la odontoides con incursión en el agujero magno y fosa posterior determinando estenosis importante del agujero magno y unión cráneo cervical. La RM (Figura 2 A-B) mostró una compresión importante de la unión bulbomedular con aparición de aumento de señal en la unión bulbomedular en relación al sitio de la estenosis y deformidad importante de esta estructura. Llamó la

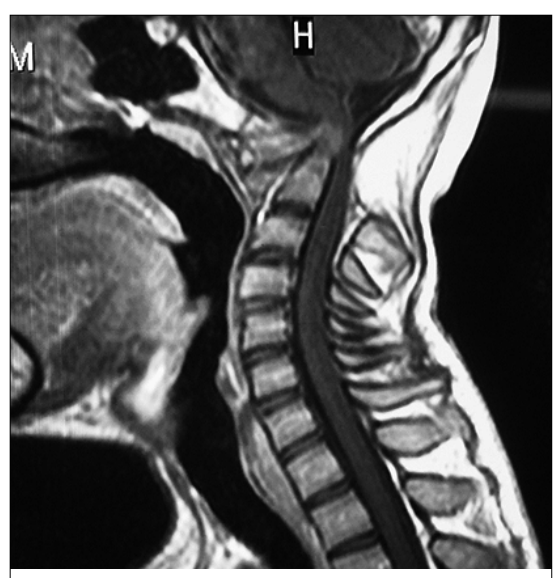

Figura 2A. Resonancia magnética vista sagital en secuencia T1 donde se visualiza desplazamiento anormal de la odontoides, interposición de tejido inflamatorio entre el clivus/arco anterior de $\mathrm{C} 1$ y la odontoides. Compresión importante de la unión bulbomedular.

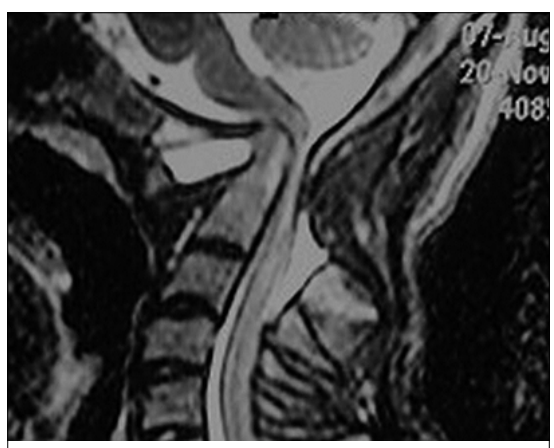

Figura 2B. RM secuencia T2 sagital donde se visualiza compresión medular con signos de mielopatía avanzados. Interposición de tejido de aspecto hiperintenso entre el clivus y la cara anterior de la odontoides. 
atención la presencia de tejido blando interpuesto entre el clivus y el ápice de la apófisis odontoides de aspecto blando sugiriendo la presencia de pannus de origen inflamatorio por la ubicación.

\section{Técnica quirúrgica}

Debido síntomas con una progresión muy rápida y drástica y el grado de compresión bulbo-medular grave desde el punto de vista radiológico decidimos colocar un halo craneal y realizar un primer abordaje quirúrgico para descomprimir la charnela occipitocervical por vía posterior, fijación occipitocervical hasta C3 a distal, con una craniectomía occipital y resección de arco posterior del atlas y plastía dural con sustituto dural con técnica de ampliación dural en bolsillo similar a la realizada en Malformación de Chiari I. Se utiliza sistema Vertex de Medtronic con monitoreo de potenciales evocados transoperatorios continuo. Se usaron Sistema de placa-barra bilaterales con tornillos a masas laterales. En un segundo tiempo 7 días posterior al primer procedimiento se realizó en primera instancia traqueostomía para manejo post-operatorio por el grado extremo de compresión de unión bulbomedular y a continuación odontoidectomía por vía anterior transoral con resección de pannus odontoideo-clival. La cabeza se fijó con cabezal Mayfield previo retiro del halo craneal. Se colocó Sistema de localización y calibración para neuronavegación Medtronic. La apertura de la cavidad oral con separadores autostáticos fijados a la mesa quirúrgica. Se realiza una incisión curva en paladar blando a derecha.

\section{Odontoidectomía}

Una vez incidida la pared posterior faríngea se usó neuronavegación para determinar la altura de resección de estructuras óseas y con motor Midas Rex se realizó resección de $2 \mathrm{~cm}$ de arco anterior del atlas en porción medial y tercio inferior del clivus, al mismo tiempo que se resecó tejido blando de aspecto fibroso color blanquecino perlado entre el borde posterior del clivus y el diente que estaba extremadamente desplazado a posterior. Una vez se comprobó ubicación de la odontoides se resecó ligamento transverso o re- manente del mismo con pinzas Kerrison de 1 y $2 \mathrm{~mm}$. Continuamos con uso del motor la odontoidectomía que se concluyó en forma total sin cambios de tipo electrofisiológico destacables durante el monitoreo. Se visualiza la duramadre con integridad de la misma en toda la zona descomprimida. Se realiza cierre por planos especialmente sutura en 2 planos de la incisión de descarga en el paladar blando y se retiraron los separadores orales.

\section{Evolución}

La evolución postquirúrgica cursó sin complicaciones, con buena evolución de la fuerza motora mejorando en un período de 7 días a $3 / 5$ en todos los grupos musculares afectados. Se retiró traqueotomía a los 7 días, se inició tratamiento de fisiatría y kinesiología todos los días permaneciendo el paciente internado por 30 días básicamente para cumplir con su período de rehabilitación inicial dentro del hospital. No hubo complicaciones desde el punto de vista infeccioso, fistula de LCR, ni dehiscencia de sutura. Presentó disfonía persistente durante 6 meses lo que atribuimos a incisión de descarga palatina. El período de seguimiento ha sido de 24 meses con recuperación de la marcha independiente, recuperación de la fuerza muscular en extremidades superiores completamente, rigidez en movimientos del cuello sin dolor local ni irradiado. Los controles radiológicos posteriores se realizaron con TAC y $\mathrm{Rx}$ funcionales que permitieron evaluar la progresión de la artrodesis (Figura $3 \mathrm{~A}$ y B).

\section{Discusión}

La invaginación basilar se refiere a una anormalidad del desarrollo que involucra una anormalidad de la columna vertebral alta que hace un prolapso en la base del cráneo ${ }^{5}$. Esta condición tiene numerosas variantes e incluye hipoplasia basioccipital, hipoplasia condilar, hypoplasia del atlas, asimilación atlanto occipital, anormalidades de odontoides, Síndrome de Klippel Feil, malformación de Chiari, siringomielia y siringobulbia ${ }^{6,7,8}$. La invaginación basilar secundaria, que habitualmente es denominada impresión basilar, es una condición asociada a reblandecimiento

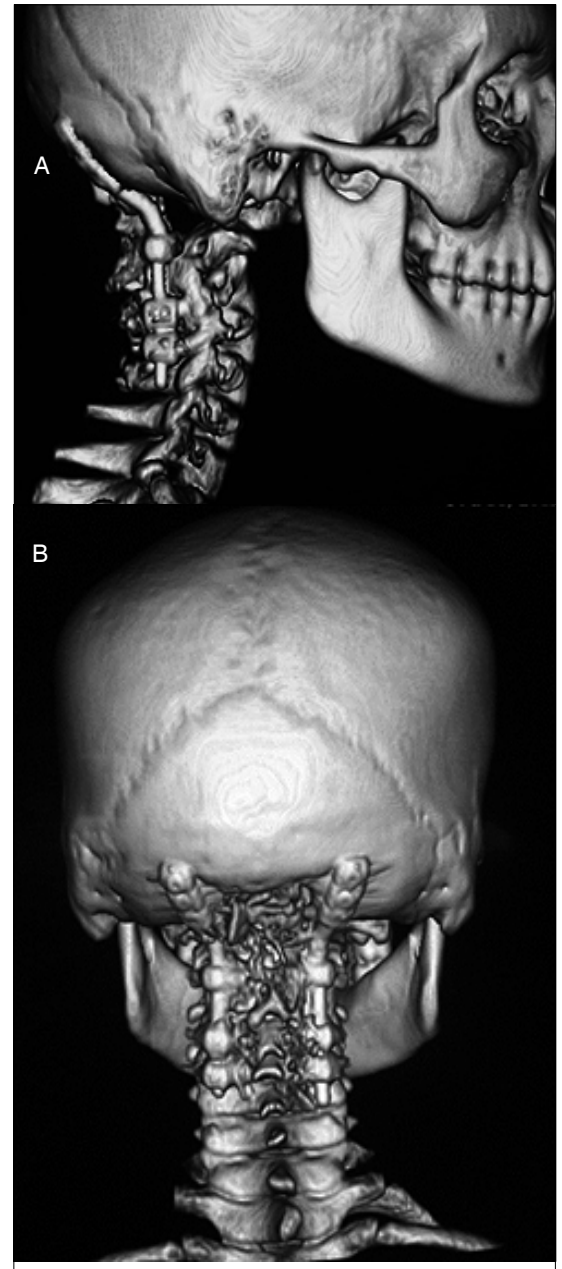

Figura $3 \mathbf{A}$ y $\mathbf{B}$. TAC post operatoria axial con reconstrucción $3 \mathrm{D}$ vista lateral y posterior de fijación y descompresión vía posterior.

de las estructuras óseas de la base del cráneo. Puede ser vista en la enfermedad de Paget, osteomalacia, osteogénesis imperfecta, osteodistrofia renal, neurofibromatosis, enfermedad de Hurler, artritis reumatoide, infecciones de la base craneal y fracturas de la base cranial posterior ${ }^{6,7,8}$. Adicionalmente la platibasia se refiere a un aplanamiento de la base craneal con un incremento del ángulo basal. Cuando es unilateral la platibasia está asociada o no con signos o síntomas. Sin embargo, la platibasia y la invaginación basilar o la impresión basilar frecuentemente coexisten $^{6,7}$.

La clasificación de Malis del año $1997^{9}$, donde la malformación de la unión craneo cervical se clasificaba en 2 grupos dependiendo de su asociación a Malformación de Chiari, en el grupo I no había asociación de Chiari, en el grupo 
II la asociación de aumento de distancia entre ondontoides y atlas y el clivus migrado superiormente al unísono postuló una disminución de volumen en la fosa posterior y por ende una herniación de las tonsilas en el canal raquídeo $^{10}$. Asímismo se puede hablar de una inestabilidad cráneo-vertebral cervical determinada por una subluxación atlanto axoidea que no desaparece con los movimientos del cuello ${ }^{11}$.

\section{Sintomatología}

Los síntomas de compresión de la unión craneo cervical pueden ser inespecíficos y de difícil localización. Esto determina que el diagnóstico muchas veces puede retrasarse y ser confundido con enfermedades desmielinizantes $\mathrm{u}$ otro tipo de patologías. Pueden desencadenarse síntomas isquémicos si hay compromiso de la arteria basilar o vertebrales, PICA o arteria espinal anterior ${ }^{5}$. En todo caso lo más frecuente es un síndrome sensitivo motor con escasa localización determinado por la compresión directa de la odontoides sobre el tronco encefálico, en lo motor es frecuente la parálisis cruzada de Bell con compromiso de ambas extremidades superiores y menor compromiso de extremidades inferiores ${ }^{12}$. Puede presentarse también alteración de los pares craneanos y además inestabilidad que se traduce en dolor cervical, neuralgia occipital, dolor radicular o agravamiento de los síntomas neurológicos ${ }^{13}$. En la asociación con siringomielia la maniobra de Valsalva, tos y estornudo tienden a empeorar una cefalea que puede ser variable. Un síndrome medular central con debilidad motora y espasticidad se pueden presentar por el compromiso de los tractos descendentes del cordón medular. Alteraciones en coordinación de la marcha puede ser atribuida a disfunción cerebelosa. Dolor en extremidades superiores e inferiores así como diplopia, disfagia y tinnitus también pueden ser descritas. Puede observarse nistagmus vertical cuando está presente habitualmente al examen dirigido y rotatorio menos frecuentemente en el plano horizontal $^{12}$.

La mayoría de hallazgos distinguibles en pacientes con malformación de Chiari II es el desplazamiento caudal del vermis cerebeloso y a través del foramen magno produciendo un "kinking" o deformidad de la unión craneovertebral. Los pacientes se presentan habitualmente en la infancia con síndorme de chiari I. La malformación de Chiari II es por lo general una disgenesia neural primaria. Los infantes pueden cursar con hidrocefalia y mielomeningocele, siringomielia y degeneración del los núcleos de los nervios cervicales bajos (se presentan con estridor, apnea, llanto débil, dificultad para alimentarse y retrocollis fijo). Los pacientes jóvenes se presentan con espasticidad, ataxia, debilidad motora, opistotonos, nistagmus y osciloopsia ${ }^{12}$. Si la segmentación entre el occipital y el atlas falla puede haber una asimilación completa o parcial del atlas y pueden asociarse a anomalías como enanismo, pectus excavatum, sindactilia, anormalidades mandibulares, hypospadias y ocasionales defectos del aparato genitourinario. Algunas de estas anormalidades pueden determinar alteraciones de flujo de la arteria vertebral con síntomas como vértigo, síncope e isquemia inclusive.

\section{Evaluación radiológica}

La articulación cráneo cervical es compleja y debe ser analizada en conjunto con todos sus elementos osteoligamentarios donde la resonancia magnética tiene un papel importante especialmente en la evaluación de los ligamentos. El agujero magno estudiado en cadáveres debe medir $34,5 \mathrm{~mm}$ (rango de 29,2 a $40,5 \mathrm{~mm}$ ) y una ancho máximo de 29,4 (rango de 26,2 a 37,0 mm) ${ }^{14}$. Existen altraciones puramente óseas como el arco hipocondilar y el tercer cóndilo, de ubicación paramediana que se articula con la adontoides a nivel del arco anterior del atlas. La hipoplasia del cóndilo condiciona una asimilación parcial o total del atlas. Frecuentemente pueden haber invaginación basilar y otras anormalidades como fusión del axis a la tercera vértebra cervical, Síndrome de Kipplel Feil y vértebra occipital con hipoplasia condilar asociados ${ }^{15,16,17}$. Las anormalidades unilaterales de las masas laterales del atlas habitualmente no están asocidas con invaginación basilar. La impresión basilar está definida por un apófisis odontoides con su ápice en un nivel superior al plano de la línea Chamberlain de por lo menos $2,5 \mathrm{~mm}$ (calculada en la vista sagital de TAC o RM y medida desde el paladar óseo al borde posterior del agujero magno). La línea de Wackenheim es la línea basal del clivus prolongada caudalmente. Normalmente la odontoides permanece anterior e inferior a ésta. La línea de McGregor empieza desde el borde posterior del paladar duro y pasa por la cortical de la concha del occipital por lo que consideramos una medición más exacta Chamberlain debido a las variaciones inclusive por grosor óseo que puede determinar Mc Gregor ${ }^{10,11}$. La línea de McRae se extiende desde el basion al borde posterior del foramen magno. La odontoides no debe proyectarse sobre esta línea. La línea bimastoidea de Fischgold la odontoides debe estar entre 3 y $10 \mathrm{~mm}$ sobre esta línea. Las anomalías óseas entre $\mathrm{C} 1 \mathrm{y}$ C2 pueden determinar fusiones unilaterales con alteración de función importante, habitualmente se asocian amalformacion de Chiari ${ }^{18}$. Chamberlain consideraba a la invaginación basilar siempre asociada a Platibasia, la posición alta de la odontoides se asociaba con una angulación horizontal y acortamiento del clivus. Klaus ${ }^{19}$ identificó 2 variantes en la invaginación basilar en relación a la línea de Wackenheim estas se clasificaban según la presencia o no de platibasia donde toma importancia la ubicación de la punta de la odontoides y su relación con la línea de Wackenheim.

La mayoría de las alteraciones óseas del axis no tiene relación con invaginación basilar, es más las más frecuentes el "os terminal" o el "os odontoideum" que es una falla de fusión del núcleo de osificación del diente conocido como osículo de Bergman, puede confundirse con una fractura de odontoides Tipo 1. Estas alteraciones se encuentran más frecuentemente asociadas a Síndrome de Morquio, mucopoliscaradosis, displasia espondiloepifisiaria congénita, displasia espondilometafisaria, enanismo metatrófico y enfermedad de Conradi $^{21,22}$. Una aplasia total de la odontoides es extremadamente rara pero asociada a una irregulariadad del borde superior del cuerpo de $\mathrm{C}^{23}$. La compresión al nivel de $\mathrm{C} 1$ puede producirse por hipoplasia y aplacia secundaria a la ausencia de las ataduras normales del ligamento apical y alares predisponiendo a una inestabilidad atlanto axoidea ${ }^{24}$.

Dentro de los criterios radiológicos debe precisarce algunas alteraciones como la distancia entre la punta de la odontoides y la unión ponto medular 
que se ve reducida en casos asociados a malformación de Chiari. El intervalo atlanto dental o clivus-dental es importante especialmente para medir o determinar la reducción especialmente con las radiografías funcionales de cuelllo. La integridad del ligamento transverso es menos importante que en lesiones traumáticas ya que por lo general la distancia o intervalo atlanto dental es mucho mayor a los $5 \mathrm{~mm}$ y hay una laxitud crónica del mismo ${ }^{25}$. Se habla de clivus anómalo cuando este está deformado o acortado, puede asociarse a platibasi e impresión basilar. La línea de Wackenheim es la línea basal del clivus prolongada caudalmente. Normalmente la odontoides permanece anterior e inferior a esta línea. La línea de Mc Rae es la línea del plano del agujero magno trazada desde el basion al opistion y habitualmente la apófisis odontoides no rebasa los límites de este plano. El retroceso odontoideo es una invaginación de la punta de la odontoides que se derige anormalmente hacia atrás, sobrepasando claramente la línea que une las clinoides posteriores al ángulo postero inferior de C2. La posición alta del diente se asociaba con una angulación horizontal y acortamiento del clivus. Klaus $^{19}$ identificó dos variantes en la invaginación en relación a la línea de Wackenheim ${ }^{6}$ éstas se diferenciaban según la presencia o no de platibasia donde toma importancia la ubicación de la punta de la odontoides y su relación con la línea de Wackenheim.

El contorno del tronco cerebral es un importante parámetro de evaluación que habitualmente no se afecta mayormente en aquellos pacientes que presentan malformación de Chiari concomitante $^{4}$.

\section{Biomecánica y fisiología}

La biomecánica primero los movimientos de la articulación craneovertebral están determinado por 2 tipos distintos, rotación (movimiento angular) y translación (movimiento lineares) ${ }^{26,27}$. Clínicamente la rotación esta dada sobre el eje $\mathrm{X}$ referida como flexión extensión, el eje rotador de y se refiere a la rotación axial, y el eje de rotación z está en referencia a los movimiento de torsión lateral. axis y rotador.

Las malformaciones congénitas de la unión craneocervical son por lo gene- ral estáticas o fijas y no pueden ser reducidas con tracción $n^{7,8,9}$. Las movimienos y alteraciones de las articulaciones merecen un estudio aparte pero al referirnos al caso que nos ocupa tenemos que considerar los efectos de una odontoidectomía transoral y su potencial efecto desestabilizador ${ }^{28}$. Está comprobado que cuando la odontoides y el ligamento transveros son removidos quirúrgicamente el rango de movimiento rotacional en $\mathrm{C} 1-\mathrm{C} 2$ se incrementa debido a un incremento a la zona laxa de la articulación. Post operatoriamente el centro de rotación angular móvil que habitualmente es no desplazable y focalizado, cambia a un centro desplazable, móvil y de distribución aumentada, esto trae como consecuencia inestabilidad traslacional y rotacional ${ }^{29}$. Aunque todos estos efectos están estudiados y medidos en laboratorio no todos los pacientes sometidos a odontoidectomía transoral van a desarrollar necesariamente una inestabilidad de este tipo ${ }^{30}$. Esto tiene que ver claramente con la asociación de fusiones preexistentes con C-0 o asimilación de las articulaciones que tienen un $50 \%$ de probabilidad de inestabilidad comparado con el $90 \%$ de posibilidades en relación a pacientes con artritis reumatoidea por ejemplo.

En 1997 Goel et al ${ }^{5}$ presentaron una clasificación basada en el análisis de 190 casos de invaginación basilar tratados por su grupo durante el período 1988-1997 basado en la presencia o no en la presencia de Chiari en grupo I y II respectivamente. En el grupo I claramente hay una invaginación de

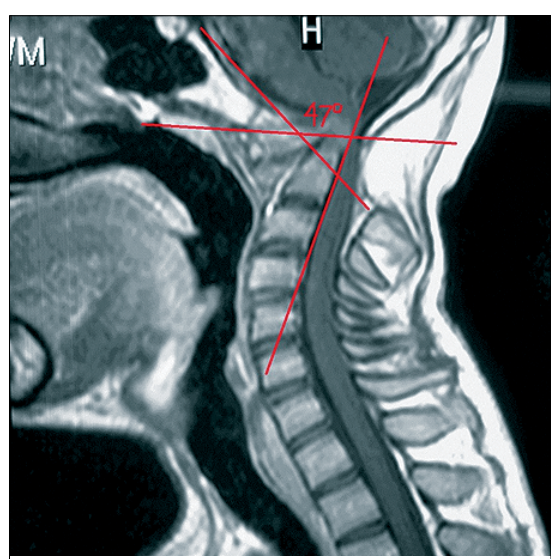

Figura 4A. Corte sagital con RM secuencia T1, en la línea media que muestra alteración del ángulo cráneo cervical de $47^{\circ}$ en pre operatorio. la odontoides en la fosa posterior y un distanciamiento considerable de la odontoides del arco anterior del atlas. En el tipo II hay digamos un clivus migrado al unísono con el arco anterior del atlas y la odontoides resultadndo en una fosa posterior de volumen reducido. La malformación de Chiari es la herniación de la amídgalas cerebelosas por la misma disminución del volumen 5. La distancia entre la odontoides y la unión pontino-bulbar fue útil para determinar la reducción de volumen de la fosa posterior y esta distancia fue marcadamente reducida en el grupo II, sin embargo, este fue relativamente grande en el grupo I. Esto lo comprobamos objetivamente mediante las mediciones de ángulo craneo vertebral que cambió de $47^{\circ}$ a $30^{\circ}$ del pre al post operatorio (Figuras $4 \mathrm{~A}$ y $4 \mathrm{~B}$ ).

El abordaje transoral de la unión craneocervical provee un acceso directo a la línea media y extraduural de esta patología. El abordaje transmucoso provee excelente descompresión del bulbo ventral y parte alta de la médula cervical, además permite acceso desde el tercio inferior del clivus hasta la vértebra C3. La exposición está determinada además por la apertura de la cavidad bucal y por los tejidos blandos como nasofaringe, orofaringe, el maxilar inferior y la base del cráneo ${ }^{32}$. El abordaje puede ser tan simple como retraer el paladar blando y la lengua sin realizar ninguna incisión adicional en estas estructuras blandas. Es un hecho que se puede producir disfagia, disfonía y regurgitación nasal de fluidos que puede ocurrir tras la incisión

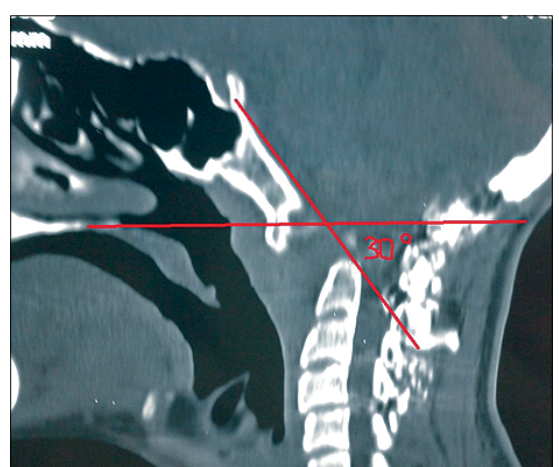

Figura 4B. TC de control postoperatoria con remoción completa de odontoides, visualización del injerto óseo paravertebral posterior, y resección del aparato ligamentario anterior y pannus inflamatorio crónico. Medición del ángulo cráneo-vertebral que disminuyó a $30^{\circ}$ comparando con los $47^{\circ}$ de la RM preop. 
del paladar o lengua. Los abordajes extendidos pueden ser el transmaxilar, transpalatal, transfacial y transmandibular y se usan más en caso de patología neoplásica. Este abordaje está recomendado para patología no reductible y compresión ventral del tronco encefálico como procedimiento inicial. En muchos casos cuando la compresión de produce sólo por tejido inflamatorio llamado pannus una fijación y artrodesis posterior puede determinanr reabsorción de la lesión compresiva y mejoría neurológica ${ }^{33,34}$. Si se decide la tracción craneal en casos de tejido inflamatorio debe optarse por un halo craneal compatible con RMN. El vector de tracción debe ser neutro o el cuello debe estar levemente extendido. Para realizar la tracción axial se debe empezar con 4 libras e ir incrementando progresivamente hasta las 10 ó 12 libras. Se debe evitar la distracción que podría provocar lesión neurológica o vascular. La flexión del cuello debe evitarse porque puede incrementar la compresión asociada a las estructuras craneales. La duración de la tracción debe limitarse a unos pocos días para evitar las úlceras de decúbito, trombosis venosa, infecciones y otras complicaciones asociadas con el reposo en cama prolongado. Una recomendación que aparece clara es la distracción manual y extensión seguida de fijación posterior la cual debe ser considerada en pacientes con invaginación basilar asociada a malformación de Chiariji,35. Si la patología compresiva es ósea o no determinada por elementos inflamatorios la descompresión y fijación interna son necesarias ${ }^{36-45}$. Desde el punto de vista práctico la odontoidectomía transforal permite una exposición extradural ventral de línea media de la unión craneo vertebral que evita manipulación de estructuras críticas como los nervios craneales, tronco encefálico, médula espinal o arterias vertebrales. En comparación al abordaje retrofaríngeo alto, los nervios craneales bajos deben ser retraídos, las ramas de la arteria carótida externa deben ser sacrificadas y la faringe debe ser movilizada extensamente. Esto puede derivar en disfagia prolongada. El abordaje retrofaríngeo tiene la relativa ventaja de ser extramucoso y es mejor para la colocación de injerto óseo y aplicación de alguna placa evitando el riesgo de contaminación bacteriana. La cirugía transoral está contraindicada en casos de infección nasofaríngea o si alguna estructura vascular por ejemplo la arteria vertebral esta relacionada íntimamente a la patología primaria.

Resumiendo el enfoque de tratamiento, existen varios algoritmos de tratamiento para malformaciones de la charnela. En este punto Dickman, Ronderos y Sonntag ${ }^{46}$ establecieron que si una malformación se reduce se debe optar por una fijación posterior. La reducción con tracción es practicada primariamente en pacientes con artritis reumatoide, infeccciones, neoplasias y enfermedades por reblandecimiento óseo ${ }^{41-47} \mathrm{Si}$ la malformación no se reduce se debe considerar si la compresión es anterior o posterior de lo cual dependerá el abordaje quirúrgico. En casos de compresión ventral y presencia de compresión por tejido inflamatorio o fibroso se debe realizar primero una descompresión posterior incluyendo C1-C2 y fijación occipito cervical. De no producirse mejoría o de persistir compresión en la imagen control idealmente con RM se debe realizar una odontoidectomía transoral. Por otro lado Goel ${ }^{5}$ propone un algoritmo dependiendo de 2 grupos. El Grupo 1 o inestable en donde la reducción o no debe ser objeto de fijación por vía posterior tanto si es fija o no. En el grupo 2 considerada estable es suficiente una descompresión posterior. En relación al abordaje posterior no hay consenso en relación al uso de plastía dural fue descrita inicialmente por Goel en $1988^{5}$.

\section{Conclusiones}

En nuestro caso el uso del neuronavegador permitió un procedimiento seguro y remoción completa del pannus inflamatorio y odontoides. La odontoidectomía anterior transoral proveee un abordaje anterior directo que evita la manipulación y maniobras sobre una zona críticamente afectada por compresión sobre el bulbo y la médula, los pares craneanos y estructuras vasculares importantes. En comparación al abordaje retrofaríngeo superior para la unión craneo cervical la ventaja sobre este abordaje es que se evita la manipulación de los nervios craneanos, donde además se sacrifican vasos ramas de la arteria carótida externa y se moviliza extensamente la faringe. Estas maniobras resultan en disfagia importante que puede ser evitada con el abordaje transoral. El abordaje retrofaríngeo tiene como ventaja el que al ser extramucoso puede permitir la colocación de injerto óseo o implantes de titanio con una tasa de infección muy baja ${ }^{18-21}$.

\section{Recibido: 28 de febrero de 2018 Aceptado: 15 de abril de 2018}

\section{Referencias}

1. Chamberlain WE, Basilar impression (platybasia). A bizarre developmental anomaly of the occipital bone and upper cervical spine with striking and misleding neurologic manifectations. Yale J Biol Med 1939; 11 (5): 487-496.

2. List CF. Neurologic syndromes accompanying developmental anomalies of occipital bone, atlas and axis. Arch neurol Psychiatry 1941 ; 45: 577-616.

3. Matson DD. Neurosurgery of infancy and childhood. Charles C Thomas 1969; 7: 119-121.

4. Royo-Salvador MB, Platibasia, impresión basilar, retroceso odontoideo y kinking del tronco cerebral, etiología común con la siringomielia, escoliosis y malformación de Arnold Chiari idiopáticas. REV NEUROL (Barc) 1996; 24 (134): 1241-1250.

5. Goel A, Bhatjiwale M, Desai K, Basilar invagination: a study based on 190 surgically treated patients. J Neurosurg 1998; 88(6):962-968.

6. Smoker WR, Khanna G. Imaging the craniocervical junction. Childs Nerv Syst 2008; 24(10): 1123-1145.

7. Hensinger RN. Congenital anomalies of the cervical spine. Clin Orthop Relat Res 1991; 264 (264): 16-38.

8. Sherman J. The craniovertebral junction. In: Rao KCVG, Williams Jp, Lee BCP, Sherman JL, eds. MRI and CT of the spine. Baltimores, MD: Williams \& Wilkins: 1994: 71-97.

9. Malis LI, Cohen I, Gross SW. Arnold Chiari malformation Arch Surg 1951; 63: 783-798. 
10. Klaus E. Roetgen diagnosis of platybasia and basilar impression: Additional experience with a new method of examination. Fortschr Geb Rontgenstr Nuklearmed 1957; 86 (4): 460-469.

11. Crockard HA, Pozo JL, Ransford AO, Stevens JM, Kendall BE,Essigman WK. Transoral decompression and posterior fusion for rheumatoid atlantoaxial subluxation. J Bone Joint Surg Br 1986; 68(3): 350-356.

12. Benglis DM, Levi AD. Neurological Findings of Craniovertebral Junction Disease.

13. Bonkowski JA, Gibson RD, Snape L. Foramen magnum meningioma: transoral resection with a bone baffle to prevent CSF leakage. Case report. J Neurosurg 1990; 72(3): 493-496.

14. de Oliveira E, Rothal R, Peace D. Microsurgical anatomy of the region of the foramen magno. Sur Neurol 1985, 24 (3): $293-352$.

15. Smoker WR, Khanna G. Imaging the craniocervical junction. Child Nerv S yst 2008, 24 (10): 1123-1145.

16. Bewermeyer H, Dreesbach HS, Hunermann B, Heiss WD. MR imaging of familial basilar impression J Comp Assit Tomogr 1984: 8 (5): 953-956.

17. Naidich TP, Mc Lone DG, Harwood-Nash DC. Malformations of the craniocervical junction In: Newton TH, Potts DG, eds. Modern Neuroradiology. Computed Tomography of the spine and spinal Cord. San Anselmo, CA: Clavadel Press; 1983: 355-366.

18. Sdherman J. The craniovertebral junction. In: Rao KCVG, Williams JP, Lee BCP, Sherman JL, eds, MRI and CT of the spine. Baltimore, MD: Williams \& Wilkins: 1994: 71-97.

19. Klaus E. Roetgen diagnosis of platybasia and basilar impression: additional experience with a new method of examination J. Fortschr Geb Rontgenstr Nuklearmed 1957; 86 (4): 460-469.

20. Menezes AH, Fenoy KA. Remnants of occipital vertebrae: proatlas segmentation abnormalities. Neurosurgery 2009, 64 (5): $945-953$, discussion 954.

21. Shohat M, Lachman R, Rimoin DL, Odontoid hypoplasia with vertebral cervical subluxation ans ventriculomegaly un metatrophic dysplasia. J Pediatr 1989: 114 (29): 239-243.

22. Thomas SL, Childress MH, Quinton B, Hypoplasia of the odontoid with atlanto-axial sunluxation in Hurler's Syndrome. Pediatri Radiol 1985; 15 (5): 353-354.

23. Van Gilder JC, Menezes AH, Dolan KD. The craniovertebral Junction and its Abnormalities. New York, NY: Futura Publishers:1987.

24. Mc Manners T. Odontoid hypolplasia. Br J Radiol 1983; 56 (672): 907-910.

25. Padget DH, Development of cranial arteries in human embryo. Contrib Embryol 1948; 32: 205-262.

26. Panjabi MM, White AA III, Brand RA Jr. A note on defining body parts configurations. J Biomech 1974; 7 (4): $385-387$

27. Goel VK, Three-dimensional motion behavior of the human spine-a question of terminology. J Biomech Eng 1987; 109(4): $353-355$.

28. Crawford NR, Songs GS, Sonntag VK, Dickman CA. Biomechanical comparison of C1-C2 posterior fixations. Cable, graft, and screw combinations. Spine 1998; 24(18): 1894-1902.

29. Naderi S, Crawford NR, Melton MS, Sonntag VK, Dickman CA. Biomechanical analysis of cranial settling after transoral odontoidectomy. Neurosurg Focus 1999; 6 (6):e7.

30. Paramore CG, Dickman CA, Sonntag VK. The anatomical suitability of the C1-C2 complex for transarticular screw fixation. J Neurosurg 1996; 85 (2): 221-224.

31. Chamberlain WE. Basilar impression (platybasia) abizarre developmental anomaly of the occipital bone and upper cervical spine with striking and misleading neurologic manifestations. Yale J Biol Med 1939; 11: 487-496.

32. Transoral Approach to the Craniovertebral Junction Curtis A. Dickman, Robert F. Spetzler, Volker K. H. Sonntag, Nicholas C. Bambakidis, and Paul J. Apostolides in Surgery of the Craniovertebral Union, Chapter 21, 277-289.

33. BouchaudChabot A, Lioté F. Cervical spine involvement in rheumatoid arthritis. A review. Joint Bone Spine 2002; 69(2): $141-154$.

34. Grob D, Schütz U, Plötz G. Occipitocervical fusion in patients with rheumatoid arthritis. Clin Orthop Relat Res 1999;366(366): 46-53.

35. Kim LJ, Rekate HL, Klopfenstein JD, Sonntag VK. Treatment of basilar invagination associated with Chiari I malformations in the pediatric population: cervical reduction and posterior occipitocervical fusion. J Neurosurg 2004; 101(2, Suppl):189-195.

36. Crockard HA, Pozo JL, Ransford AO, Stevens JM, Kendall BE, Essigman WK. Transoral decompression and posterior fusion for rheumatoid atlantoaxial subluxation. J Bone Joint Surg Br 1986; 68(3): 350-356.

37. Menezes AH, VanGilder JC. Transoraltranspharyngeal approach to the anterior craniocervical junction. Tenyear experience with 72 patients. J Neurosurg 1988; 69(6): 895-903.

38. Crockard HA. The transoral approach to the base of the brain and upper cervical cord. Ann R Coll Surg Engl 1985;67(5):321-325.

39. Masferrer R, Hadley MN, Bloomfield S, et al. Transoral microsurgical resection of the odontoid process. BNI Q 1985;1(3):34-40.

40. Spetzler RF, Selman WR, Nash CL Jr, Brown RH. Transoral microsurgical odontoid resection and spinal cord monitoring. Spine 1979; 4(6): 506-510.

41. Bonkowski JA, Gibson RD, Snape L. Foramen magnum meningioma: transoral resection with a bone baffle to prevent CSF leakage. Case report. J Neurosurg 1990; 72(3): 493-496.

42. Hadley MN, Spetzler RF, Sonntag VKH. The transoral approach to the superior cervical spine. A review of 53 cases of extradural cervicomedullary compression. J Neurosurg 1989; 71(1): 16-23.

43. Menezes AH, VanGilder JC, Graf CJ, McDonnell DE. Craniocervical abnormalities. A comprehensive surgical approach. J Neurosurg 1980; 53(4): 444-455 14. Mullan S, Naunton R, HekmatPanah J, Vailati G. The use of an anterior approach to ventrally placed tumors in the foramen magnum and vertebral column. J Neurosurg 1966; 24(2): 536-543.

44. Scoville WB, Sherman IJ. Platybasia, report of 10 cases with comments on familial tendency, a special diagnostic sign, and the end results of operation. Ann Surg 1951; 133(4): 496-502.

45. Spetzler RF, Dickman CA, Sonntag VKH. The transoral approach to the anterior cervical spine. Contemp Neurosurg. 1991;13(9):1-6.

46. Dickman CA, Ronderos JF, Sonntag VKH. Stabilization of the craniovertebral junction in rheumatoid arthritis. Part I: Pathophysiology, diagnosis and surgical criteria. Contemp Neurosurg 1995; 17[12]: 1-6.

47. Mullan S, Naunton R, Hekmat Panah J, Vailati G. The use of an anterior approach to ventrally placed tumors in the foramen magnum and vertebral column. J Neurosurg 1966; 24(2): 536-543

\section{Correspondencia a:}

José Vicente Vasconez Fabre

Institución: Hospital del Profesor - Serv. Neurocirugía.

Dirección: Alameda 4860, Santiago

Fono:(56.2) 2299.6300

josevasconez04@gmail.com 